\title{
NUTRIENTES NA SEIVA DE PLANTAS CÍTRICAS FERTIRRIGADAS ${ }^{1}$
}

\author{
THAIS REGINA DE SOUZA², ROBERTO LYRA VILLAS BÔAS ${ }^{3}$, \\ JOSÉ ANTÔNIO QUAGGIO ${ }^{4}$, LEANDRO CAIXETA SALOMÃO
}

RESUMO - A análise de nutrientes na seiva vem destacando-se como ferramenta sensível no estudo do estado nutricional de plantas perenes, sendo útil também na avaliação da disponibilidade de nutrientes no solo para as plantas. Este experimento foi conduzido com o objetivo de avaliar a concentração de nutrientes na seiva de duas variedades de laranja, quando fornecidas cinco doses de $\mathrm{N}, \mathrm{P}_{2} \mathrm{O}_{5}$ e $\mathrm{K}_{2} \mathrm{O}$ via fertirrigação. A seiva foi extraída mensalmente do ramo da brotação nova com éter etílico, durante duas safras consecutivas. Para compreender a dinâmica dos nutrientes na seiva, também foram feitas, simultaneamente, análises de folha e de solução do solo. Os valores de $\mathrm{pH}$ na seiva mantiveram-se constantes, em torno de 5,5, independentemente dos tratamentos. A análise da seiva mostrou-se sensível às variações da adubação, pois as concentrações de $\mathrm{N}$ e K na seiva aumentaram conforme as doses de nutrientes aplicadas e correlacionaram-se de forma positiva com os valores desses nutrientes na folha e na solução do solo. Alta concentração de $\mathrm{K}$ foi observada na seiva, em torno de $4,0 \mathrm{~g} \mathrm{~L}^{-1}$, sendo esta maior que todos os outros nutrientes analisados. Observou-se diferença significativa no teor de $\mathrm{N}_{-} \mathrm{NO}_{3}$ entre as duas variedades de copa estudadas: a variedade de copa Hamlin, de ciclo precoce, apresentou concentração na seiva cerca de $20 \%$ superior à variedade Valência, que é de ciclo tardio. Os resultados sugerem que a análise da seiva pode ser empregada como ferramenta auxiliar na avaliação do estado nutricional de plantas cítricas.

Termos para indexação: análise da seiva, análise foliar, solução do solo.

\section{NUTRIENTS IN THE SAP OF FERTIGATED CITRUS PLANTS}

\begin{abstract}
The analysis of nutrients in the sap has been gaining attention as a sensitive tool for the study of the nutritional state of perennial plants, being also useful in assessing the availability of nutrients for the plants in the soil. This study was conducted with the purpose of evaluating the concentration of nutrients in the sap of two varieties of sweet orange trees, when supplied with five rates of $\mathrm{N}_{2} \mathrm{P}_{2} \mathrm{O}_{5}$ and $\mathrm{K}_{2} \mathrm{O}$ via fertigation. The sap was monthly extracted from stems of new shoots with ethyl ether during two consecutive growth seasons. In order to understand the dynamics of the nutrients in the sap, analyses of leaf and soil solution were also made at the same time. The $\mathrm{pH}$ in the sap remained constant at around 5.5, regardless the treatments. The sap analysis showed to be very sensitive to variations in the fertilization regime, because the concentrations of $\mathrm{N}$ and $\mathrm{K}$ in the sap increased with the applied rates of nutrients, and also because it had a positive correlation with the values of these nutrients in leaf and soil solution. A high concentration of $\mathrm{K}$ was observed in the sap, at about $4.0 \mathrm{~g} \mathrm{~L}^{-1}$, being higher than the concentration of all the other nutrients. There were significant differences in the $\mathrm{N}^{-\mathrm{NO}_{3}}$ level between the two studied varieties: the Hamlin variety, with early maturity, showed $\mathrm{N}-\mathrm{NO}_{3}$ concentration in the sap at about $20 \%$ higher than the Valencia variety, which is late maturity. The results suggest that the sap analysis can be used as tool in assessing the nutritional status of citrus plants.
\end{abstract}

Index terms: sap analysis, leaf analysis, soil solution.

\footnotetext{
1(Trabalho 171-11). Recebido em: 06-06-2011. Aceito para publicação em: 29-03-2012. Trabalho extraído de Tese de Doutorado. Suporte financeiro Fapesp.

${ }^{2}$ Pós-Doutoranda do Instituto Agronômico de Campinas (IAC), Centro de Solos e Recursos Ambientais. Avenida Barão de Itapura 1481, CEP: 13012-970, Campinas-SP, e-mail: tr_souza@yahoo.com.br

${ }^{3}$ Professor Doutor do Departamento de Recursos Naturais/Ciência do Solo, da Faculdade de Ciências Agronômicas FCA/UNESP. Rua José Barbosa de Barros 1780, CEP: 18610-307, Botucatu-SP, e-mail: rlvboas@fca.unesp.br

${ }^{4}$ Pesquisador do IAC, Centro de Solos e Recursos Ambientais, e-mail: quaggio@iac.sp.gov.br

${ }^{5}$ Doutorando do Curso de Pós-Graduação em Irrigação e Drenagem, FCA/UNESP, e-mail: 1salomao@fca.unesp.br
} 


\section{INTRODUÇÃO}

A área irrigada na citricultura paulista cresceu de modo acelerado nos últimos anos, alcançando, em 2009, cerca de 125 mil hectares, o que representa $21 \%$ da área cultivada com citros (COELHO, 2010). A maior parte dessa área emprega o sistema de irrigação localizada por gotejo, associado à técnica de fertirrigação, que consiste na aplicação simultânea de fertilizantes com a água através do sistema de irrigação, cuja principal vantagem é o ganho de eficiência de uso dos nutrientes, devido ao maior parcelamento da adubação, o que reduz as perdas por lixiviação e, especialmente, pelo melhor ajuste entre a demanda e o suprimento de nutrientes, nos diferentes estádios de desenvolvimento da planta (QUAGGIO et al., 2006).

Como a técnica de fertirrigação permite aplicações frequentes de nutrientes, é importante desenvolver critérios diagnósticos mais sensíveis às variações da disponibilidade de nutrientes no solo e da demanda por nutrientes pelas plantas de modo a ajustar a quantidade de fertilizantes aplicada e, assim, evitar perdas por falta ou excesso de nutrientes, garantindo maior eficiência na adubação. Isso pode ser feito através do monitoramento da concentração de nutrientes na solução do solo, como também através da análise da seiva, que é adequada para determinar o estado nutricional atual da planta. De acordo com Cadahía e Lucena (2005), a composição da seiva pode variar por um grande número de fatores: hora do dia, órgão amostrado e sua posição na planta, adubação, condições climáticas e outros, e, portanto, deve ser usada, principalmente, em avaliações nutricionais pontuais.

O trabalho de Moreno e Garcia-Matinez (1983) demonstrou que a análise da seiva pode ser muito útil para a compreensão do metabolismo de espécies de plantas lenhosas, pois extraíram seiva do xilema de ramos maduros de laranjeiras Washington Navel e verificaram que cerca de $98 \%$ do nitrogênio contido nos vasos xilemáticos estava na forma de compostos aminados e aminoácidos, e apenas $2 \%$ na forma mineral. Isso confirma os resultados de Bar-Akiva e Sagiv (1967) que demonstraram que os citros têm alta atividade de redutase do nitrato, porém não se sabe se o baixo teor de nitrato na seiva é devido ao fato de as folhas receberem o nitrogênio na forma reduzida, ou se o nitrato é rapidamente metabolizado nas folhas.

As variações de demanda por nutrientes na videira, durante os diferentes estádios fenológicos, foram acompanhadas através da diagnose foliar e pela análise da seiva, extraída do pecíolo da planta, em um ensaio de fertirrigação conduzido por Cadahía et al. (2005). Os autores verificaram que a análise da seiva foi mais sensível às variações da adubação, principalmente com potássio, refletindo melhor os resultados de produção, o que permitiu melhorar a qualidade dos frutos.

Nesse contexto, este experimento foi elaborado com o objetivo de avaliar a concentração de nutrientes na seiva de duas variedades de laranja, quando fornecidas cinco doses de $\mathrm{N}, \mathrm{P}_{2} \mathrm{O}_{5}$ e $\mathrm{K}_{2} \mathrm{O}$ via fertirrigação.

\section{MATERIAL E MÉTODOS}

O experimento foi desenvolvido no município de Reginópolis, Estado de São Paulo, na fazenda Emu, pertencente à empresa Citrovita/Votorantim, com as variedades de copa Valência e Hamlin, ambas sobre o porta-enxerto citrumelo Swingle. O projeto foi instalado em uma área onde as duas variedades de laranja estavam próximas, portanto estas ficaram no mesmo setor de fertirrigação. O espaçamento de plantio foi de $7 \times 4 \mathrm{~m}$, o experimento foi conduzido por duas safras consecutivas, 2007/2008 e 2008/2009, quando as plantas possuíam, respectivamente, 5 e 6 anos de idade.

No início do experimento, o solo, a 0-20 $\mathrm{cm}$ de profundidade e a $20 \mathrm{~cm}$ da linha de emissores, possuía as seguintes características químicas, segundo os métodos de análise descritos em Raij et al. (2001): 5,0 de $\mathrm{pH}\left(\mathrm{CaCl}_{2}\right) ; 9 \mathrm{~g} \mathrm{dm}^{-3}$ de matéria orgânica; $57 \mathrm{mg} \mathrm{dm}^{-3}$ de P (resina); 22; 3,0; 14; 5; 22 e $44 \mathrm{mmol}_{\mathrm{c}} \mathrm{dm}^{-3}$ de $\mathrm{H}+\mathrm{Al}, \mathrm{K}, \mathrm{Ca}, \mathrm{Mg}$, soma de bases (SB) e capacidade de troca de cátions (CTC), respectivamente, e $50 \%$ de saturação por bases. A textura do solo de $0-60 \mathrm{~cm}$ de profundidade foi classificada como arenosa, com teores de areia, silte e argila de 814; 57 e $132 \mathrm{~g} \mathrm{~kg}^{-1}$, respectivamente (teor de argila inferior a $15 \%$ ). O solo foi classificado como Argissolo Vermelho-Amarelo (EMBRAPA, 2006).

Os tratamentos foram constituídos por cinco doses de $\mathrm{N}, \mathrm{P}_{2} \mathrm{O}_{5}$ e $\mathrm{K}_{2} \mathrm{O}$, aplicadas via fertirrigação. A dose completa (100\%) foi definida com base no histórico de análises de solo e folha, e também em função da produtividade esperada do talhão, e correspondeu a 140; 40 e $130 \mathrm{~kg} \mathrm{ha}^{-1} \mathrm{ano}^{-1}$, respectivamente, de N, $\mathrm{P}_{2} \mathrm{O}_{5}$ e $\mathrm{K}_{2} \mathrm{O}$ (QUAGGIO et al., 2005). Portanto, foram estabelecidos os seguintes tratamentos: T1- apenas irrigado (sem nutrientes); T2-fertirrigado com $25 \%$ da dose de $\mathrm{N}, \mathrm{P}_{2} \mathrm{O}_{5}$ e $\mathrm{K}_{2} \mathrm{O}$; T3-fertirrigado com $50 \%$ da dose de N, $\mathrm{P}_{2} \mathrm{O}_{5}$ e $\mathrm{K}_{2} \mathrm{O}$; T4-fertirrigado com $100 \%$ da dose de $\mathrm{N}, \mathrm{P}_{2} \mathrm{O}_{5}$ e $\mathrm{K}_{2} \mathrm{O}$; e T5-fertirrigado com $200 \%$ da dose de N, $\mathrm{P}_{2} \mathrm{O}_{5}$ e $\mathrm{K}_{2} \mathrm{O}$.

As fontes de $\mathrm{N}, \mathrm{P}_{2} \mathrm{O}_{5}$ e $\mathrm{K}_{2} \mathrm{O}$ utilizadas foram, respectivamente, nitrato de amônio, ácido fosfórico 
e cloreto de potássio branco. Os micronutrientes B, Mn e Zn também foram aplicados via fertirrigação, nas formas de ácido bórico, sulfato de manganês e sulfato de zinco, porém em doses iguais para todos os tratamentos. As plantas foram fertirrigadas entre os meses de setembro a abril de cada safra, duas vezes por semana, durante 34 semanas, totalizando 68 aplicações. Apenas o fósforo foi aplicado no início da safra, entre os meses de setembro e novembro. O período de estresse, período em que as plantas não recebem água e nutrientes para promover indução floral, foi de maio a julho, aproximadamente. No mês de agosto, as plantas foram somente irrigadas.

As parcelas experimentais foram constituídas por três linhas com doze plantas, sendo que as dez plantas da linha central foram usadas nas avaliações das variáveis de interesse do experimento. As duas linhas laterais e as duas plantas nos extremos da linha central formavam a bordadura, totalizando 36 árvores por parcela. Todo o experimento ocupava uma área de aproximadamente 4 ha. O delineamento estatístico empregado foi o de blocos ao acaso, com quatro repetições.

Quanto ao sistema de irrigação, as parcelas possuíam linhas simples de tubos gotejadores, instaladas ao lado do tronco das árvores, paralelamente à linha de plantio, com emissores autocompensantes espaçados entre si a 0,6 m, formando uma faixa molhada contínua, com largura máxima de $0,8 \mathrm{~m}$, e os emissores forneciam uma vazão de $2,3 \mathrm{~L} \mathrm{~h}^{-1}$ e trabalhavam com pressão de serviço entre 5 a 30 mca. A injeção dos fertilizantes foi feita através do injetor venturi. $\mathrm{O}$ manejo da irrigação foi realizado com base na medida diária da evaporação, através do tanque Classe A, obtendo-se a evapotranspiração potencial (ETP) e a evapotranspiração da cultura (ETc), conforme Allen et al. (1998). O experimento também foi monitorado por tensiometria, sendo instaladas três baterias de tensiômetros por variedade, e cada bateria possuía tensiômetros nas profundidades de 0,$3 ; 0,6$ e $0,9 \mathrm{~m}$.

A produção foi quantificada considerando-se a massa e o número de frutos. O cálculo da produção foi feito a partir da multiplicação do número de frutos por planta pela massa média dos frutos, obtendo assim a produção em $\mathrm{kg}$ planta ${ }^{-1}$. Considerando o número de plantas por área, obteve-se a produção em $\mathrm{t} \mathrm{ha}^{-1}$. De cada parcela, foi determinado o número total de frutos de cinco plantas.

\section{Extração da seiva (solução extraída com éter etílico denominada de seiva)}

Para obter a seiva, foram coletados 40 ramos da brotação nova por parcela, considerando-se as 10 plantas úteis, retirou-se um ramo por quadrante, na altura mediana da planta, no período da manhã, e no dia seguinte a irrigação/fertirrigação das plantas. Os ramos sem as folhas foram acondicionados em sacos de papel e levados ao laboratório em caixas de isopor com bolsa térmica. A seiva foi analisada, segundo a metodologia descrita por Cadahía e Lucena (2005), e adaptada por Souza (2010).

Durante as duas safras, com início em setembro de 2007 e término em agosto de 2009 , foram realizadas 22 coletas de ramos da brotação nova, uma por mês, sempre na segunda quinzena, exceto nos meses de outubro de 2007 e fevereiro de 2009 . No período de estresse a coleta também foi realizada, independentemente da irrigação/fertirrigação.

No laboratório, os ramos foram limpos com gaze umedecida em água destilada, secos sobre papel toalha, cortados em frações de 1 a $2 \mathrm{~cm}$ e acondicionados em frascos plásticos de boca larga que receberão éter etílico padrão analítico. A quantidade de éter utilizada por amostra era o suficiente para cobrir os ramos, e assim garantir a completa extração da seiva. Como a extração foi feita em material fresco, no máximo um dia após a coleta, os ramos já estavam no éter e eram imediatamente congelados pelo período aproximado de 15 dias, em freezer convencional, com temperatura próxima de $-15^{\circ} \mathrm{C}$.

Depois do descongelamento, as amostras foram transferidas para funis de separação, para separar os pigmentos vegetais dissolvidos em éter do extrato de seiva, que não são miscíveis. A seiva, por ser mais densa, ficava situada na parte inferior do funil. Os 40 ramos coletados proporcionavam, aproximadamente, de 2 a $5 \mathrm{ml}$ de extrato de seiva, dependendo da época de coleta.

A quantificação dos nutrientes $\mathrm{K}, \mathrm{P}, \mathrm{Ca}, \mathrm{Mg}$, $\mathrm{S}, \mathrm{B}, \mathrm{Cu}, \mathrm{Fe}, \mathrm{Mn}$ e $\mathrm{Zn}$, contidos no extrato de seiva, foi realizada através da leitura direta em espectrômetro de plasma de argônio, sem filtragem, e com diluição de 10 vezes. $O$ nitrogênio, nas formas de $\mathrm{N}-\mathrm{NH}_{4}$ e N-NO${ }_{3}$, foi determinado por destilação a vapor, sem diluição, segundo a metodologia descrita por Raij et al. (2001).

A solução extraída com éter etílico não era apenas seiva, e como os ramos foram congelados, as células romperam, extravasando o suco celular. Portanto, a solução extraída era seiva mais conteúdo celular.

\section{Coleta das folhas e da solução do solo}

As folhas foram retiradas nos quatro quadrantes das 10 plantas úteis de cada parcela, e sempre foram amostrados dois quadrantes por planta de forma alternada, sendo coletadas a $3^{\mathrm{a}}$ e a $4^{\mathrm{a}}$ folhas 
recém-maduras, a partir de um ramo dreno, que possuía fruto ou brotação nova, totalizando 40 folhas por parcela. Após a coleta, as folhas foram acondicionadas em sacos de papel e levadas ao laboratório para análise, conforme a metodologia descrita por Bataglia et al. (1983). Foram realizadas 22 coletas de folhas nos mesmos dias de coleta dos ramos da brotação nova para análise da seiva.

Para extrair a solução do solo, foi instalado um extrator de solução na região central de cada parcela, na profundidade de $30 \mathrm{~cm}$, a $20 \mathrm{~cm}$ de um emissor e sob a projeção da copa das plantas. O extrator de solução do solo era composto de tubo de PVC, conectado na extremidade inferior a uma cápsula de cerâmica porosa. $\mathrm{Na}$ extremidade superior, o extrator era vedado com rolha, e por esta passavam dois tubos de plástico com diâmetro de aproximadamente dois $\mathrm{mm}$, em um deles se aplicava o vácuo e no outro ficava conectada uma seringa por onde se coletava a solução.

Aproximadamente 12 horas após a fertirrigação, era aplicado nos extratores vácuo de $80 \mathrm{kPa}$, com o auxílio de uma bomba de vácuo manual, e cerca de 6 horas após este procedimento, era feita a coleta da solução. Foram feitas extrações de solução do solo nos meses de novembro de 2007, janeiro, fevereiro, março, abril, outubro e novembro de 2008 e janeiro, março, abril e maio de 2009, totalizando 11 coletas. Na solução do solo extraída, foram determinados os valores de $\mathrm{pH}$, nitrogênio mineral $\left(\mathrm{N}^{-\mathrm{NH}_{4}}\right.$ e N-NO ${ }_{3}$ ), $\mathrm{P}, \mathrm{K}, \mathrm{Ca}, \mathrm{Mg}, \mathrm{Mn}$ e $\mathrm{Zn}$ diretamente, sem filtragem ou digestão, segundo os métodos descritos por Raij et al. (2001).

Os resultados obtidos foram submetidos à análise de variância (ANOVA - Teste F a 5\% e 1\% de probabilidade), aos estudos de correlação e a testes de regressão (linear e polinomial) e comparação de médias (Tukey a $5 \%$ de probabilidade), utilizando o programa estatístico SISVAR (FERREIRA, 2003).

\section{RESULTADOS E DISCUSSÃO}

\section{Valores de pH na solução do solo e na seiva}

$\mathrm{O}$ pH da solução do solo diminuiu com o aumento da dose de nutrientes. Essa variação ocorreu, de maneira bastante similar, na área das duas variedades de copa estudadas, chegando a atingir valores médios em torno de 3,5 no tratamento T5 (Tabela 1). Como a fertirrigação foi feita via irrigação por gotejo, desta forma, o fertilizante ficava concentrado na zona do bulbo molhado, que é um volume de solo restrito, e as fontes de nitrogênio e fósforo utilizadas, respectivamente, nitrato de amô- nio e ácido fosfórico, possuem caráter ácido.

A acidificação observada na solução do solo prejudicou o processo de nitrificação, e a concentração de $\mathrm{N}-\mathrm{NH}_{4}$ na solução aumentou, principalmente nas doses mais elevadas, nos tratamentos T4 e T5 (Tabela 1), o que afetou também a concentração de $\mathrm{N}_{-} \mathrm{NH}_{4}$ na seiva, que foi mais alta nos tratamentos que receberam as maiores doses de fertilizantes (Tabela 2). Diferente da concentração de $\mathrm{N}^{-\mathrm{NO}_{3}}$ na solução que aumentou gradualmente do tratamento $\mathrm{T} 1$ ao tratamento $\mathrm{T} 5$, sendo o mesmo comportamento observado na seiva.

A maior absorção de amônio pela planta provoca desequilíbrio no balanço de cátions e ânions absorvidos, forçando a planta a sintetizar mais ácidos orgânicos e, posteriormente, exsudar prótons $\left(\mathrm{H}^{+}\right)$ pelas raízes para manter o equilíbrio iônico dentro das células. Esse processo, além de provocar maior acidificação do solo, reduz a absorção de outros cátions, como o Ca e o Mg. Estes nutrientes na solução do solo apresentaram comportamento inverso ao da seiva, em função dos tratamentos, principalmente para a variedade de copa Valência (Figura 1). Holzschuh et al. (2009) observaram que a presença de amônio na solução nutritiva afetou negativamente os teores de Ca no tecido de plantas de arroz, porém não teve efeito sobre a absorção de $\mathrm{N}, \mathrm{Mg}$ e K.

O excesso de amônio na solução do solo pode ser muito prejudicial para a cultura da laranja, pois uma das particularidades das plantas cítricas é o fato de a concentração de Ca ser muito alta em todos os tecidos, superior à de outros nutrientes, incluindo o nitrogênio, com exceção dos frutos. Esta característica não é comum a outras culturas (MATTOS JUNIOR et al., 2005). De acordo com Quaggio et al. (2005), nas folhas geradas na primavera com seis meses de idade e de ramos com frutos, a concentração adequada de $\mathrm{N}$ varia de 23 a $27 \mathrm{~g} \mathrm{~kg}^{-1}$ enquanto a de $\mathrm{Ca}$ varia de 35 a $45 \mathrm{~g} \mathrm{~kg}^{-1}$.

Dou et al. (1999) demonstraram que o fornecimento contínuo de amônio causou redução do crescimento de mudas de citrumelo Swingle e tangerina Cleópatra, comparado ao fornecimento de nitrato. Estes autores verificaram sintomas de toxicidade de amônio a concentrações maiores que $15 \mathrm{mg} \mathrm{L}^{-1}$ na solução, que foram associados a injúrias na estrutura dos cloroplastos e acúmulo de amido e fitoferritina, causando prejuízos ao sistema fotossintético. O tratamento $\mathrm{T} 5$, que atingiu valores de $\mathrm{pH}$ próximos a 3,5, apresentou concentrações de amônio na solução do solo que chegaram a $60 \mathrm{mg} \mathrm{L}^{-1}$ (Tabela 1).

A despeito da redução acentuada nos valores de pH da solução do solo, em função das doses de fertilizantes aplicadas na fertirrigação, os valores de $\mathrm{pH}$ na seiva mantiveram-se praticamente constantes 
ao longo de todo o período experimental, com valores de 5,4 e 5,5, respectivamente, para as variedades Valência e Hamlin (Tabela 2). Esses valores de pH estão próximos aos valores normalmente encontrados nos vacúolos das células vegetais, que é geralmente ácido ao redor de 5,5, segundo Taiz e Zeiger (2004). Esses autores mencionam ainda que, em células vegetais maduras, o vacúolo ocupa geralmente $90 \%$ ou mais do volume celular. Parte do extrato de seiva obtido no presente trabalho é suco celular, pois a extração foi feita em ramos da brotação nova, que, quando congelados, romperam as células, expondo o conteúdo dos vacúolos.

\section{Concentração dos nutrientes na seiva}

Encontrou-se alta concentração de K na seiva, sendo esta maior que todos os outros nutrientes analisados na forma mineral, diferente da análise foliar que apresenta teores de $\mathrm{Ca}$ e $\mathrm{N}$ maiores que o de $\mathrm{K}$ (Tabelas 2 e 3). Este nutriente tem como funções principais a ativação de enzimas e o controle osmótico, e permanece na planta, principalmente na forma iônica (MALAVOLTA, 2006). O teor de K na seiva apenas apresentou diferença significativa, em relação aos tratamentos, para a variedade Valência. Apesar de o teste $\mathrm{F}$ não ter sido significativo para a variedade de copa Hamlin a 1 e $5 \%$, foi significativo a 6,5\% (Tabela 2). Alta correlação foi observada entre o teor de $K$ na seiva e o teor de $\mathrm{K}$ na folha, respectivamente, $0,87^{*} \mathrm{e}$ $0,85^{*}$, para as variedades de copa Valência e Hamlin.

O teor de P na seiva somente apresentou diferença significativa em relação aos tratamentos para a variedade de copa Valência. De modo geral, foram observados altos valores de $\mathrm{P}$ em todos os tratamentos, mesmo no tratamento T1, que não recebeu nutriente, o que se deve à menor adsorção de $\mathrm{P}$ à superfície dos óxidos hidratados de ferro e alumínio, em função dos maiores valores de $\mathrm{pH}$ observados na solução do solo, nos tratamentos que receberam baixas doses de $\mathrm{P}$ (Tabela 1). Além disso, o solo inicialmente possuía teor muito alto de $\mathrm{P}\left(57 \mathrm{mg} \mathrm{dm}^{-3}\right)$, o que certamente contribuiu para os valores elevados de $\mathrm{P}$ observados na seiva do tratamento T1. A concentração de P na folha apresentou pouca variação em função dos tratamentos, em ambas as variedades (Tabela 3 ). Os valores de P na seiva não se correlacionaram de forma positiva com os valores de $\mathrm{P}$ foliar, e a concentração de P na solução do solo foi maior nos tratamentos que receberam maiores doses de nutrientes (Tabela 1).

Os teores de nitrogênio mineral $\left(\mathrm{N}^{-\mathrm{NH}_{4}}+\right.$ $\mathrm{N}-\mathrm{NO}_{3}$ ) na seiva são muito menores do que os teores de potássio e de fósforo. Entretanto, vale lembrar que o nitrogênio nesta forma representa menos de $5 \%$ do
$\mathrm{N}$ total contido na seiva da laranjeira, segundo Moreno e Garcia-Martinez (1983). Isso ocorre devido à alta taxa de redução de nitrato dos cítricos, conforme foi discutido por Bar-Akiva e Sagiv (1967).

$\mathrm{O}$ teor de nitrato e amônio na seiva aumentou de acordo com as doses de $\mathrm{N}, \mathrm{P}_{2} \mathrm{O}_{5}$ e $\mathrm{K}_{2} \mathrm{O}$ aplicadas, apresentando variação significativa entre os tratamentos para as duas variedades estudadas (Tabela 2). $\mathrm{O}$ teor de nitrogênio nas folhas apresentou a mesma variação observada na seiva (Tabela 3 ). Correlações estreitas foram observadas entre as concentrações $\mathrm{N}$ na seiva e na folha, respectivamente, $0,96 * *$ e $0,86^{*}$ para as variedades Valência e Hamlin.

A análise da seiva mostrou-se bastante sensível às doses de nutrientes aplicados, pois com exceção de $\mathrm{P}$, cuja disponibilidade já era alta no solo, as concentrações de $\mathrm{N}$ e $\mathrm{K}$ aumentaram com $\mathrm{o}$ aumento nas doses de $\mathrm{N}, \mathrm{P}_{2} \mathrm{O}_{5}$ e $\mathrm{K}_{2} \mathrm{O}$ aplicadas. Observou-se também que, aumentando as doses de fertilizantes, ocorreram aumentos nas concentrações de nutrientes na solução do solo com reflexos na concentração deles na seiva, pois correlações estreitas foram observadas entre essas variáveis (Tabela 4).

Outro dado importante está relacionado à amplitude dos valores encontrados. Enquanto a concentração de $\mathrm{N}$ na seiva variou de 84,4 a $157,8 \mathrm{mg} \mathrm{L}^{-1}$ entre os tratamentos, considerando as duas variedades, o teor de $\mathrm{N}$ na folha variou de 26 a $30 \mathrm{~g} \mathrm{~kg}^{-1}$. Para o $\mathrm{P}$ e K os valores na seiva variaram de 3,8 a 4,2 e de 3,3 a $3,6 \mathrm{~g} \mathrm{~L}^{-1}$, respectivamente, e na folha foram de 13 a 17 e de 1,3 a $1,4 \mathrm{~g} \mathrm{~kg}^{-1}$. Portanto, a seiva refletiu melhor os tratamentos aplicados, principalmente no caso do nitrogênio, mostrando a sensibilidade da técnica de análise.

As concentrações dos micronutrientes $\mathrm{Mn}$ e $\mathrm{Zn}$ na seiva, para as duas variedades de copa estudadas, aumentaram conforme as doses de nutrientes aplicadas (Tabela 2). A disponibilidade destes nutrientes aumenta com a diminuição dos valores de $\mathrm{pH}$ (ABREU et al., 2007). A acidificação observada na solução do solo proporcionou maior disponibilidade destes nutrientes (Tabela 1) e, consequentemente, os mesmos foram absorvidos em maiores quantidades pelas plantas, comportamento semelhante foi encontrado para os resultados da análise foliar (Tabela 3 ).

As concentrações de Mn na seiva e na folha correlacionaram-se de forma positiva e apresentaram valores de $\mathrm{R}^{2}$ iguais a $0,99 * *$ e $0,98 * *$ para as variedades de copa Valência e Hamlin, respectivamente. $\mathrm{O}$ mesmo foi observado para o teor de $\mathrm{Zn}$ na seiva e na folha com valores de correlação de $0,81 *$ para ambas as variedades de copa.

$\mathrm{O}$ valores de $\mathrm{B}, \mathrm{Cu}$ e Fe na seiva e na folha, apesar de terem apresentado diferenças siginificati- 
vas para o teste $\mathrm{F}$ em relação aos tratamentos aplicados, exceto o $\mathrm{Cu}$ na variedade de copa Valência e o Fe na variedade de copa Hamlin, não variaram de forma coerente com os tratamentos (Tabelas 2 e 3). Comportamento semelhante foi observado para o $\mathrm{S}$, que na seiva apresentou diferença significativa em função dos tratamentos apenas para a variedade de copa Hamlin (Tabela 2) e na folha não foi observada diferença significativa em ambas as variedades (Tabela 3).

\section{Diferenças entre variedades e valores de produção}

Quando se comparam as duas variedades, maiores valores de $\mathrm{N}_{-} \mathrm{NO}_{3}$ e $\mathrm{Cu}$ foram observados na seiva da variedade de copa Hamlin e maior teor de Fe foi encontrado na variedade Valência, para todos os outros nutrientes avaliados, o teste de Tukey a $5 \%$ de probabilidade não apresentou diferença significativa (Tabela 2).

A variedade de copa Hamlin, de ciclo precoce, apresentou concentração de $\mathrm{N}_{-} \mathrm{NO}_{3}$ na seiva cerca de $20 \%$ superior à variedade Valência, que é de ciclo tardio (Tabela 2). Pozzan e Tribone (2005) comentaram que, após a abertura das flores e a queda das pétalas, a velocidade de transformações bioquímicas dos frutos é maior nas cultivares precoces. A variedade de copa Hamlin, por ser bastante produtiva e de ciclo precoce, apresenta maior exigência nutricional pontual, em um menor espaço de tempo, em relação à variedade de copa Valência que é de ciclo tardio. Portanto, a necessidade nutricional entre as variedades de laranja foi distinta. Na análise foliar, este comportamento entre variedades também foi observado (Tabela 3 ).

A produtividade na safra de 2008/2009, após dois anos de desenvolvimento do experimento, apresentou ajuste quadrático em função das doses de N, $\mathrm{P}_{2} \mathrm{O}_{5}$ e $\mathrm{K}_{2} \mathrm{O}$ aplicadas via fertirrigação, para ambas as variedades (Figura 2A). Nas funções ajustadas, os valores máximos de produção foram de 39 e $33 \mathrm{t}$ ha $^{-1}$, que corresponderam a 135 e $138 \%$ da dose de $\mathrm{N}, \mathrm{P}_{2} \mathrm{O}_{5}$ e $\mathrm{K}_{2} \mathrm{O}$ aplicada, respectivamente, para as laranjeiras Valência e Hamlin. Os valores de produção foram baixos considerando sistemas fertirrigados, isso provavelmente ocorreu devido à acidificação observada na solução do solo, gerada pelos tratamentos aplicados (Tabela 1). Os baixos valores de $\mathrm{pH}$ encontrados nos tratamentos que receberam as maiores doses de $\mathrm{N}, \mathrm{P}_{2} \mathrm{O}_{5}$ e $\mathrm{K}_{2} \mathrm{O}$ alteraram a dinâmica dos nutrientes na solução do solo, refletindo de forma direta na planta (Tabelas 2 e 3) e, consequentemente, na produção.
Nas condições em que este experimento foi desenvolvido, buscou-se definir o valor adequado de $\mathrm{N}$ e K na seiva em função da produção e de acordo com as equações obtidas nas Figuras 2B, 3A e 3B. $\mathrm{O}$ teor de $\mathrm{N}_{-} \mathrm{NO}_{3}$ na seiva, para as duas variedades de laranja, apresentou comportamento diferente em função da produção. Para a variedade de copa Valência, a relação $\mathrm{N}_{-} \mathrm{NO}_{3}$ na seiva versus produção apresentou ajuste quadrático, e na função ajustada, o maior valor de $\mathrm{N}_{-} \mathrm{NO}_{3}$ na seiva foi de $96,8 \mathrm{mg} \mathrm{L}^{-1}$, que correspondeu à produção de $39 \mathrm{t} \mathrm{ha}^{-1}$. Para a laranjeira Hamlin, o ajuste foi linear, portanto não foi possível obter um valor máximo, indicando que maiores teores de nitrato na seiva poderiam gerar maiores produções. O valor máximo de $\mathrm{N}-\mathrm{NO}_{3}$ observado na seiva da laranjeira Hamlin foi $131,8 \mathrm{mg}$ $\mathrm{L}^{-1}$ no tratamento $\mathrm{T} 4$.

Como os teores de $\mathrm{N}_{-} \mathrm{NH}_{4}$ e de $\mathrm{K}$ na seiva foram semelhantes entre as duas variedades (Tabela 2), a interpretação dos resultados foi realizada utilizando valores médios. Os teores de $\mathrm{N}_{-} \mathrm{NH}_{4}$ e $\mathrm{K}$ apresentaram ajuste quadrático em função da produção, e na função ajustada, o valor máximo de $\mathrm{N}-\mathrm{NH}_{4}$ e K corresponderam a 26,9 $\mathrm{mg} \mathrm{L}^{-1}$ e $4,1 \mathrm{~g} \mathrm{~L}^{-1}$, respectivamente (Figuras $3 \mathrm{~A}$ e $3 \mathrm{~B}$ ). $\mathrm{O}$ teor de 26,9 $\mathrm{mg} \mathrm{L}^{-1}$ de amônio na seiva pode ser indicativo do valor máximo tolerado pela cultura sem perdas de produtividade. A mesma relação foi feita para o teor de P na seiva, porém os resultados não apresentaram correlação significativa com a produção. 

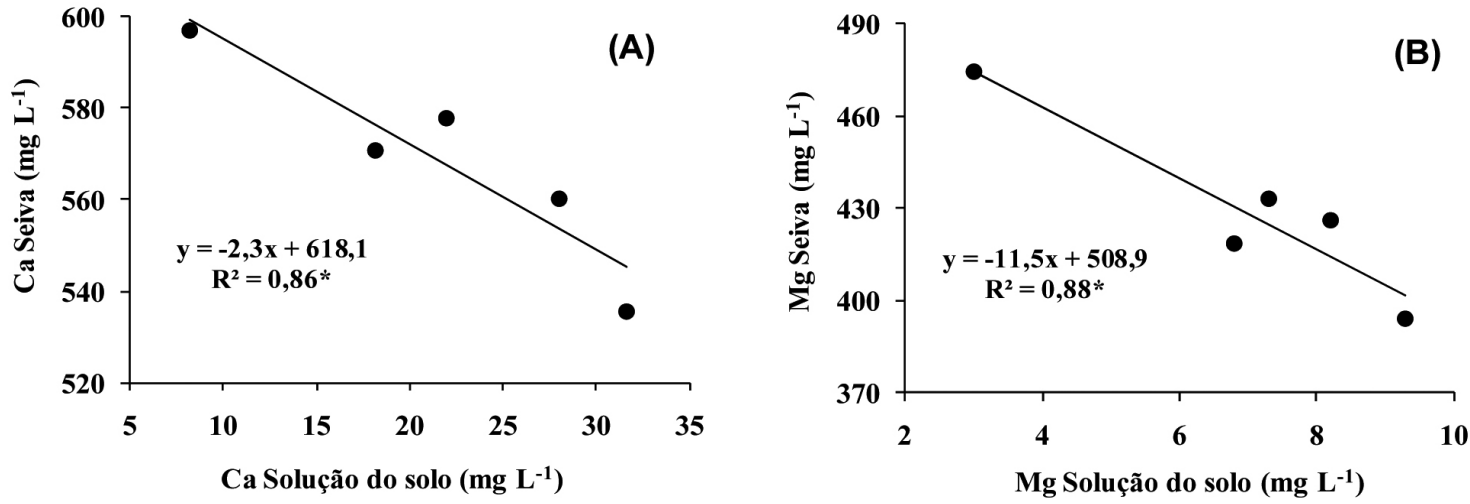

FIGURA 1 - Relação entre a concentração de Ca e Mg na solução do solo (30 cm de profundidade) e na seiva, para a variedade de copa Valência: (A) cálcio e (B) magnésio.
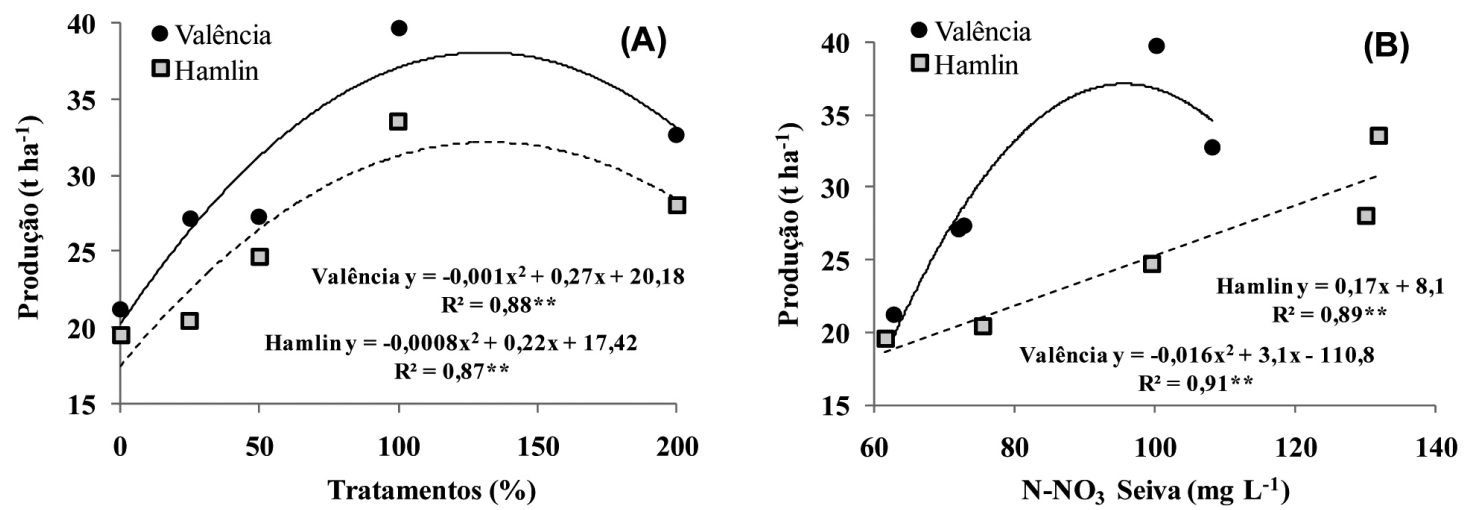

FIGURA 2 - Valores de produção (A) e relação entre a concentração de $\mathrm{N}-\mathrm{NO}_{3}$ na seiva e a produção, para as duas variedades de laranja.
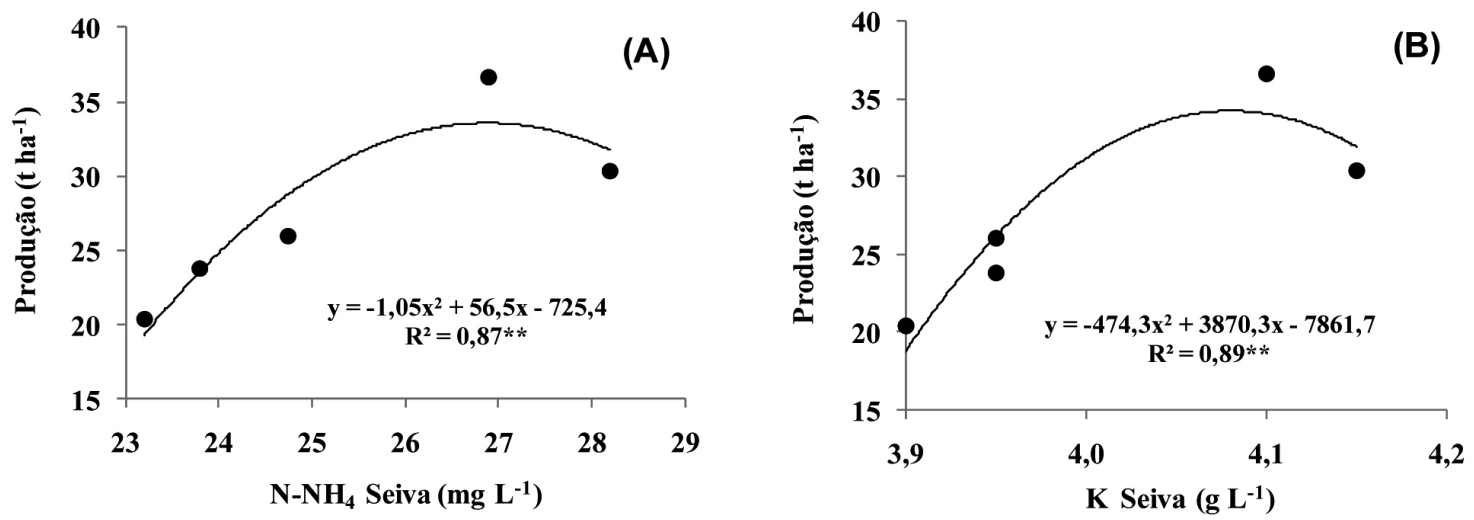

FIGURA 3 - Relação entre a concentração de $\mathrm{N}-\mathrm{NH}_{4}(\mathrm{~A})$ e $\mathrm{K}(\mathrm{B})$ na seiva e a produção (valores médios para as duas variedades de laranja). 
TABELA 1 - Valores de pH e concentração de nutrientes na solução do solo, a $30 \mathrm{~cm}$ de profundidade, em função dos tratamentos, para as laranjeiras 'Valência' e 'Hamlin'.

\begin{tabular}{|c|c|c|c|c|c|c|c|c|c|}
\hline Tratamento & pH & N-NH 4 & $\mathrm{~N}-\mathrm{NO}_{3}$ & $\mathbf{P}$ & $\mathbf{K}$ & $\mathrm{Ca}$ & Mg & Mn & Zn \\
\hline \multicolumn{10}{|l|}{ Valência } \\
\hline $\mathrm{T} 1(0 \%)$ & 5,7 & 7,5 & 6,9 & 1,3 & 9,1 & 8,2 & 3,0 & 1,0 & 1,6 \\
\hline T2 (25\%) & 5,2 & 7,1 & 13,6 & 1,2 & 13,5 & 18,2 & 7,3 & 1,9 & 2,5 \\
\hline T3 (50\%) & 3,8 & 7,7 & 34,9 & 1,1 & 40,5 & 28,0 & 8,2 & 5,2 & 5,5 \\
\hline T4 (100\%) & 3,6 & 26,9 & 78,5 & 2,6 & 62,8 & 31,6 & 9,3 & 6,8 & 6,6 \\
\hline T5 (200\%) & 3,3 & 67,3 & 102,9 & 8,5 & 122,0 & 22,0 & 6,8 & 7,0 & 7,6 \\
\hline Teste F (Trat.) & $117,9 * *$ & $40,4 * *$ & $78,2 * *$ & $36,3 * *$ & $97,3 * *$ & $15,4 * *$ & $7,1 * *$ & $34,3 * *$ & $24,3 * *$ \\
\hline Regressão & $\mathbf{Q} * *$ & $\mathbf{L} * *$ & $\mathbf{Q} * *$ & $\mathbf{Q} * *$ & $\mathbf{L} * *$ & $\mathbf{Q} * *$ & $\mathbf{Q} * *$ & $\mathbf{Q} * *$ & $\mathbf{Q} * *$ \\
\hline $\mathbf{R}^{2}$ & 0,93 & 0,94 & $\mathbf{0 , 9 7}$ & 0,99 & 0,99 & $\mathbf{0 , 9 7}$ & 0,91 & 0,96 & 0,95 \\
\hline CV (\%) & 15,2 & 116,4 & 66,1 & 119,1 & 62,3 & 71,5 & 86,0 & 71,7 & 73,3 \\
\hline \multicolumn{10}{|l|}{ Hamlin } \\
\hline T1 (0\%) & 6,1 & 6,4 & 11,5 & 2,3 & 7,6 & 5,5 & 1,3 & 0,5 & 1,5 \\
\hline $\mathrm{T} 2(25 \%)$ & 5,2 & 8,5 & 22,6 & 1,8 & 20,1 & 15,0 & 5,9 & 2,5 & 2,6 \\
\hline T3 (50\%) & 4,6 & 7,7 & 31,1 & 2,6 & 28,4 & 18,0 & 7,5 & 4,2 & 4,1 \\
\hline T4 (100\%) & 4,0 & 23,3 & 52,9 & 8,6 & 58,1 & 20,4 & 5,5 & 4,5 & 3,5 \\
\hline T5 (200\%) & 3,6 & 59,8 & 97,4 & 13,4 & 85,7 & 28,1 & 5,2 & 4,9 & 5,0 \\
\hline Teste F (Trat.) & $90,3 * *$ & $46,1 * *$ & $52,9 * *$ & $22,5 * *$ & $40,0 * *$ & $22,5 * *$ & $16,6 * *$ & $19,6 * *$ & $9,8 * *$ \\
\hline Regressão & $\mathbf{Q} * *$ & $\mathbf{L} * *$ & $\mathbf{L} * *$ & $\mathbf{L} * *$ & $\mathbf{L} * *$ & $\mathbf{L} * *$ & $\mathbf{Q} * *$ & $\mathbf{Q} * *$ & $\mathbf{L} * *$ \\
\hline $\mathbf{R}^{2}$ & 0,99 & 0,94 & 0,99 & 0,93 & 0,97 & 0,86 & 0,54 & 0,93 & 0,75 \\
\hline CV $(\%)$ & 14,8 & 104,7 & 71,8 & 108,2 & 82,9 & 66,2 & 73,7 & 81,2 & 83,2 \\
\hline
\end{tabular}

$(* *)$ significativo a $1 \%,(*)$ significativo a $5 \%,\left({ }^{n s}\right)$ não significativo, $\mathrm{L}$ - ajuste linear, $\mathrm{Q}$ - ajuste quadrático e Trat. $=$ Tratamento.

TABELA 2 - Relação entre a concentração de N, K, Mn e Zn na solução do solo (30 cm de profundidade) e na seiva, para as variedades de copa Valência e Hamlin.

\begin{tabular}{|c|c|c|c|}
\hline Variedade & Nutriente & $\begin{array}{c}\text { Equação } \\
\mathrm{y}=\text { seiva e } \mathrm{x}=\text { solução do solo }\end{array}$ & Coeficiente de Determinação $\left(\mathbf{R}^{2}\right)$ \\
\hline Valência & NT & $y=0,3 x+85,1$ & $0,95 * *$ \\
\hline Valência & $\mathrm{K}$ & $y=0,002 x+3,9$ & $0,72^{(\mathrm{ns}-7 \%)}$ \\
\hline Valência & $\mathrm{Mn}$ & $y=0,5 x+0,3$ & $0,90 *$ \\
\hline Valência & $\mathrm{Zn}$ & $y=0,3 x+2,3$ & $0,96 * *$ \\
\hline Hamlin & NT & $y=-0,01 x^{2}+1,1 x+47,1$ & $0,98 * *$ \\
\hline Hamlin & $\mathrm{K}$ & $y=0,003 x+3,8$ & $0,97 * *$ \\
\hline Hamlin & $\mathrm{Mn}$ & $y=0,35 x^{2}+1,1 x+1,5$ & $0,93 * *$ \\
\hline Hamlin & $\mathrm{Zn}$ & $y=0,7 x+1,6$ & $0,72 *$ \\
\hline
\end{tabular}

$\mathrm{NT}=$ nitrogênio total $\left(\mathrm{NH}_{4}+\mathrm{NO}_{3}\right),(* *)$ significativo a $1 \%,(*)$ significativo a $5 \%$, (ns - 7\%) não significativo a $1 \%$ e a $5 \%$, significativo a $7 \%$. 


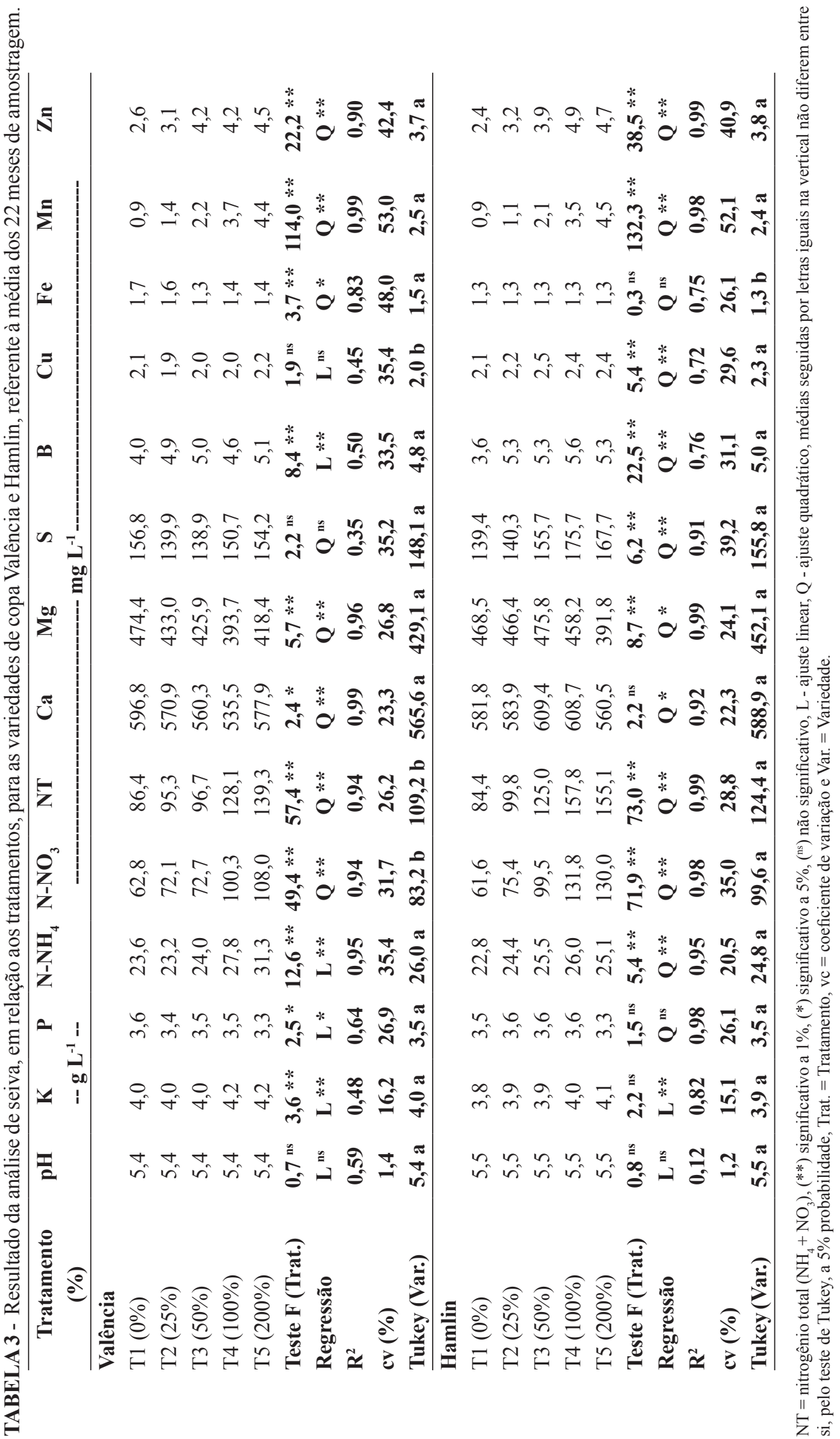


TABELA 4 - Resultado da análise foliar, em relação aos tratamentos, para as variedades de copa Valência e Hamlin, referente à média dos 22 meses de amostragem.

\begin{tabular}{|c|c|c|c|c|c|c|c|c|c|c|c|}
\hline Tratamento & $\mathbf{N}$ & $\mathbf{P}$ & $\mathbf{K}$ & $\mathrm{Ca}$ & Mg & $\mathbf{S}$ & B & $\mathrm{Cu}$ & $\mathrm{Fe}$ & Mn & $\mathrm{Zn}$ \\
\hline$(\%)$ & - & - & $\mathrm{g} \mathrm{k}_{\mathbf{s}}$ & $g^{-1}--$ & - & 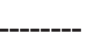 & - & 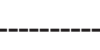 & $---m g ~ k g$ & -1 ------- & ----- \\
\hline \multicolumn{12}{|l|}{ Valência } \\
\hline $\mathrm{T} 1(0 \%)$ & 26 & 1,3 & 13 & 36 & 3,8 & 3,0 & 120 & 33 & 188 & 79 & 41 \\
\hline T2 $(25 \%)$ & 26 & 1,3 & 13 & 36 & 3,7 & 3,0 & 173 & 34 & 174 & 113 & 44 \\
\hline T3 (50\%) & 27 & 1,3 & 14 & 34 & 3,6 & 3,0 & 178 & 33 & 166 & 143 & 51 \\
\hline T4 (100\%) & 28 & 1,4 & 15 & 34 & 3,4 & 3,0 & 176 & 33 & 183 & 206 & 50 \\
\hline $\mathrm{T} 5(200 \%)$ & 28 & 1,3 & 14 & 35 & 3,4 & 3,0 & 162 & 33 & 180 & 238 & 45 \\
\hline Teste F (Trat.) & $49,9 * *$ & $2,5 *$ & $15,5 * *$ & $9,1 * *$ & $22,6 * *$ & $0,7^{\text {ns }}$ & $54,1 * *$ & $0,6^{\mathrm{ns}}$ & $16,4 * *$ & $151,7 * *$ & $2,9 *$ \\
\hline Regressão & $\mathbf{Q}^{* *}$ & $Q^{*}$ & $\mathbf{Q}^{* *}$ & $\mathbf{Q}^{* *}$ & $\mathbf{Q} * *$ & $\mathbf{L}^{\mathrm{ns}}$ & $\mathbf{Q}^{* *}$ & $\mathbf{L}^{\mathrm{ns}}$ & $\mathbf{Q}^{* *}$ & $\mathbf{Q} * *$ & $\mathbf{Q} * *$ \\
\hline $\mathbf{R}^{2}$ & 0,98 & 0,63 & 0,94 & 0,67 & 0,99 & 0,31 & 0,71 & 0,54 & 0,19 & 0,99 & 0,76 \\
\hline cv (\%) & 5,4 & 7,9 & 9,9 & 9,2 & 10,7 & 8,0 & 19,7 & 17,4 & 11,3 & 32,9 & 52,2 \\
\hline Tukey (Var.) & $27 \mathrm{~b}$ & $1,3 \mathrm{~b}$ & $14 \mathrm{~b}$ & 35 a & 3,6 a & $3,0 \mathrm{a}$ & $162 \mathrm{~b}$ & 33 a & $178 \mathrm{~b}$ & $156 \mathrm{a}$ & $46 \mathrm{~b}$ \\
\hline \multicolumn{12}{|l|}{ Hamlin } \\
\hline $\mathrm{T} 1(0 \%)$ & 26 & 1,4 & 15 & 34 & 3,3 & 3,1 & 125 & 32 & 194 & 93 & 45 \\
\hline T2 (25\%) & 27 & 1,4 & 16 & 34 & 3,2 & 3,0 & 208 & 30 & 200 & 94 & 49 \\
\hline Т3 (50\%) & 27 & 1,4 & 16 & 34 & 3,2 & 3,1 & 203 & 29 & 192 & 137 & 51 \\
\hline T4 (100\%) & 28 & 1,4 & 16 & 35 & 3,3 & 3,1 & 209 & 28 & 199 & 184 & 53 \\
\hline T5 (200\%) & 30 & 1,4 & 17 & 33 & 2,8 & 3,1 & 190 & 30 & 196 & 223 & 56 \\
\hline Teste F (Trat.) & $138,4 * *$ & $2,3^{\mathrm{ns}}$ & $14,9 * *$ & $11,7 * *$ & $30,7 * *$ & $2,3^{\mathrm{ns}}$ & $122,4 * *$ & $4,1 * *$ & 1,9 ns & $101,8 * *$ & $19,1 * *$ \\
\hline Regressão & $\mathbf{Q}^{* *}$ & $\mathbf{Q}^{\mathrm{ns}}$ & $\mathbf{L} * *$ & $\mathbf{Q}^{* *}$ & $\mathbf{Q} * *$ & $\mathbf{Q}^{*}$ & $\mathbf{Q}^{* *}$ & $\mathbf{Q} * *$ & $\mathbf{Q}^{\mathrm{ns}}$ & $\mathbf{Q} * *$ & $\mathbf{Q} * *$ \\
\hline $\mathbf{R}^{2}$ & 0,99 & 0,09 & $\mathbf{0 , 8 3}$ & $\mathbf{0 , 8 0}$ & 0,91 & 0,61 & 0,66 & 0,90 & 0,05 & 0,97 & 0,98 \\
\hline cv (\%) & 4,1 & 7,5 & 9,2 & 7,3 & 10,1 & 8,6 & 16,5 & 25,0 & 12,2 & 37,0 & 18,3 \\
\hline Tukey (Var.) & $28 \mathrm{a}$ & $1,4 a$ & $16 \mathrm{a}$ & $34 \mathrm{~b}$ & $3,1 \mathrm{~b}$ & $3,1 \mathrm{a}$ & $187 a$ & $30 \mathrm{~b}$ & $196 \mathrm{a}$ & $146 \mathrm{a}$ & 51 a \\
\hline
\end{tabular}

$(* *)$ significativo a $1 \%,(*)$ significativo a $5 \%,\left({ }^{\mathrm{ns}}\right)$ não significativo, $\mathrm{L}$ - ajuste linear, Q - ajuste quadrático, médias seguidas por letras iguais na vertical não diferem entre si, pelo teste de Tukey, a 5\% probabilidade, Trat. $=$ Tratamento, cv $=$ coeficiente de variação e Var. $=$ Variedade.

\section{CONCLUSÕES}

1-A análise da seiva é sensível às variações da adubação com $\mathrm{N}$ e $\mathrm{K}$.

2-Os valores de $\mathrm{pH}$ na seiva mantém-se constantes, independentemente dos tratamentos e da acidificação observada na solução do solo.

3-Existe alta concentração de $\mathrm{K}$ na seiva, sendo esta maior que todos os outros nutrientes minerais analisados.

4-Maior teor de $\mathrm{N}_{-} \mathrm{NO}_{3}$ é encontrado na seiva da variedade de copa Hamlin, quando comparada à variedade de copa Valência.

5-O teor de $27 \mathrm{mg} \mathrm{L}^{-1}$ de $\mathrm{N}-\mathrm{NH}_{4}$ na seiva da laranjeira pode ser indicativo do valor limiar tolerado pela cultura.

6-A análise da seiva pode ser empregada como ferramenta auxiliar na avaliação do estado nutricional de plantas cítricas.

\section{AGRADECIMENTOS}

À Fundação de Amparo à Pesquisa do Estado de São Paulo (FAPESP), pela concessão de bolsa e auxílio à pesquisa; ao Instituto Agronômico de Campinas (IAC), pelo apoio durante as análises, e à empresa Citrovita - Votorantim/Agroindústria, pela contribuição e participação no desenvolvimento deste ensaio.

\section{REFERÊNCIAS}

ABREU, C. A.; LOPES, A. S.; SANTOS, G. C. G. Micronutrientes. In: NOVAIS, R. F.; ALVAREZ, V. H.; BARROS, N. F.; FONTES, R. L. F.; CANTARUTTI, R. B.; NEVES, J. C. Fertilidade do solo. Viçosa: Sociedade Brasileira de Ciência do Solo, 2007. p. 645-763. 
ALLEN, R. G.; PEREIRA, L. S.; RAES, D.; SMITH, M. Crop evapotranspiration: gidelines for computing crop water requirements. Roma: FAO, 1998. 300p. (Irrigation and Drainage Paper, 56).

BATAGLIA, O. C.; FURLANI, A. M. C.; TEIXEIRA, J. P. F.; FURLANI, P. R.; GALO, J. R. Métodos de análise química de plantas. Campinas: Instituto Agronômico, 1983. 48p. (Circular, 78).

BAR-AKIVA, A.; SAGIV. Nitrate reductase in the citrus plants: properties, assay conditions and distributions within the plant. Physiologia Plantarum, Copenhagem, v. 20, p. 500-506, 1967.

CADAHÍA, C.; LUCENA, J. J. Diagnostico de nutrición y recomendaciones de abonado. In: ADAHÍA, C. Fertirrigación: cultivos hortícolas, frutales y ornamentales. 3. ed. Madrid: Ediciones Mundi-Prensa, 2005. p. $183-257$.

CADAHÍA, C.; MARTÍN, I.; SENTIS, J. A. Fetirrigación racional da la vid. In: CADAHÍA, C. Fertirrigación: cultivos hortícolas, frutales y ornamentales. 3. ed. Madrid: Ediciones Mundi-Prensa, 2005. p. 603-623.

COELHO, R. B. Irrigação por gotejo: problemas e soluções. Citricultura Atual, Cordeirópolis, n.76, p.12-14, 2010.

DOU, H.; ALVA, A. K.; BONDADA, B. R. Growth and chloroplast ultrastructure of two citrus rootstock seedlings in response to ammonium an nitrate nutrition. Journal Plant of Nutrition, New York, v.22, p.1731-1744, 1999.

EMBRAPA - Empresa Brasileira De Pesquisa Agropecuária. Sistema brasileiro de classificação de solos. 2.ed. Rio de Janeiro: Embrapa Solos, 2006. 306p.

FERREIRA, D. F. Sisvar versão 4.2. Lavras: DEX/ UFLA, 2003

HOLSCHUH, M. J.; BOHNEN, H.; ANGHINONI, I.; MEURER, E. J.; CARMONA, F. C.; COSTA, S. E. V. G. A. Resposta do arroz irrigado ao suprimento de amônio e nitrato. Revista Brasileira de Ciência do Solo, Campinas, v. 33, p. 1323-1331, 2009.
MALAVOLTA, E. Manual de nutrição mineral de plantas. São Paulo: Editora Agronômica Ceres, 2006. 638p.

MATTOS JÚNIOR, D.; BATAGLIA, O. C.; QUAGGIO, J. A. Nutrição dos citros. In: MATTOS JÚNIOR, D.; DE NEGRI., J. D.; PIO, R. M.; POMPEU JUNIOR, J. Citros. Campinas: Instituto Agronômico e Fundag, 2005. p. 197-219

MORENO, J.; GARCÍA-MATÍNEZ, J. L. Seasonal variation nitrogenous in the xylem sap citrus. Physiologia Plantarum, Copenhagen, v. 59, p. 669-675, 1983.

POZZAN, M.; TRIBONI, H. R. Colheita e qualidade do fruto. In: MATTOS JÚNIOR, D.; DE NEGRI. J. D.; PIO, R. M.; POMPEU JÚNIOR, J. Citros. Campinas: Instituto Agronômico e Fundag, 2005. p. 801-822.

QUAGGIO, J. A.; MATTOS JÚNIOR, D.; CANTARELLA, H. Manejo da fertilidade do solo na citricultura. In: MATTOS JÚNIOR, D.; DE NEGRI., J. D.; PIO, R. M.; POMPEU JÚNIOR, J. Citros. Campinas: Instituto Agronômico e Fundag, 2005. p. 483 - 507.

QUAGGIO, J. A.; SILVA, G. O.; SOUZA, T. R.; MATTOS JÚNIOR, D.; MENEZES, G.; KRONTAL, Y. Dinâmica de íons no solo e ganhos de eficiência fertilizante devido à irrigação e fertirrigação na citricultura. In: REUNIÃO BRASILEIRA DE FERTILIDADE E MICROBIOLOGIA DO SOLO, 2006 , Bonito. Anais... CD-ROM.

RAIJ, B. V.; ANDRADE, J. C.; CANTARELLA, H.; QUAGGIO, J. A. Análise química para avaliação da fertilidade de solos tropicais. Campinas: Instituto Agronômico, 2001. 285p.

SOUZA, T R. Monitoramento do estado nutricional de plantas cítricas e da solução do solo em sistema de fertirrigação. 2010. 131f. Tese (Doutorado em Agronomia/Irrigação e Drenagem) - Faculdade de Ciências Agronômicas, Universidade Estadual Paulista, Botucatu, 2010

TAIZ, L.; ZEIGER, E. Fisiologia vegetal. 3.ed. Porto Alegre: Artmed, 2004. 719p. 